\title{
Prolonged survival following intrapleural rupture of a dissecting aortic aneurysm
}

\author{
R. LANCASTER \\ M.R.C.P. \\ Central Middlesex Hospital, London, N.W.10
}

RUPTURE is the usual cause of death in cases of dissecting aneurysm of the aorta in the acute stage. In Sheenan's series of 300 cases (1934) the pericardium was the commonest site of rupture, the pleural cavity being next in frequency. Sheenan described only two cases that survived intrapleural rupture of a dissecting aneurysm, one for 17 days, the other 19 days, and in a review of 505 cases of dissecting aneurysm in the English literature from 1934 to 1958 , Hirst, Johns \& Kine (1958) describe only six cases that survived for 1 month, of which one survived for 3 months.

Post (1941) described the case of a 51-year-old man with multiple sclerosis who survived the rupture of a dissecting aneurysm into his left pleura for 9 weeks before dying from a septicaemia, the consequence of bed sores. Necropsy showed laminated clot at the site of rupture and Post suggested that the leak had been a slow one allowing the patient to survive the rupture. Campbell (1946) described a case of ruptured dissecting aneurysm who survived for 3 months and was shown at necropsy to have died from further rupture.

In this case report, the patient remains alive and well 16 months after rupture of a dissecting aneurysm into the left pleural cavity.

\section{Case report}

The patient, a 77-year-old woman, who had been treated with reserpine for hypertension for 12 years, was admitted to hospital on 4 May 1965 with a history of chest pain. The pain had started suddenly, 10 days prior to admission and was of agonizing severity initially, radiating all over her body but with maximum severity in her chest. She was treated at home by her doctor with oral analgesics and bed rest. The pain eased in severity after a few hours but remained as a dull ache for the 10 days before admission.

On examination she appeared well and cheerful. She was clinically anaemic ; pulse 90 regular, blood pressure $200 / 115 \mathrm{mmHg}$ equal in both arms; there were signs of left ventricular enlargement, no aortic diastolic murmur and no heart failure. There was a large left pleural effusion. Chest X-ray showed a massive left pleural effusion. Her haemoglobin was $62 \%$. ECG showed left ventricular hypertrophy. WR negative.

From the left pleural cavity was aspirated $1100 \mathrm{ml}$ of heavily blood stained fluid. Chest X-ray following aspiration showed an aneurysm of the descending thoracic aorta (Fig. 1). X-ray of the abdomen showed calcified abdominal aorta with no signs of aneurysmal dilatation.

\section{Progress}

She was treated with bed rest for 1 month and mobilized during a further month in hospital. She had two further attacks of chest pain, one 1 week

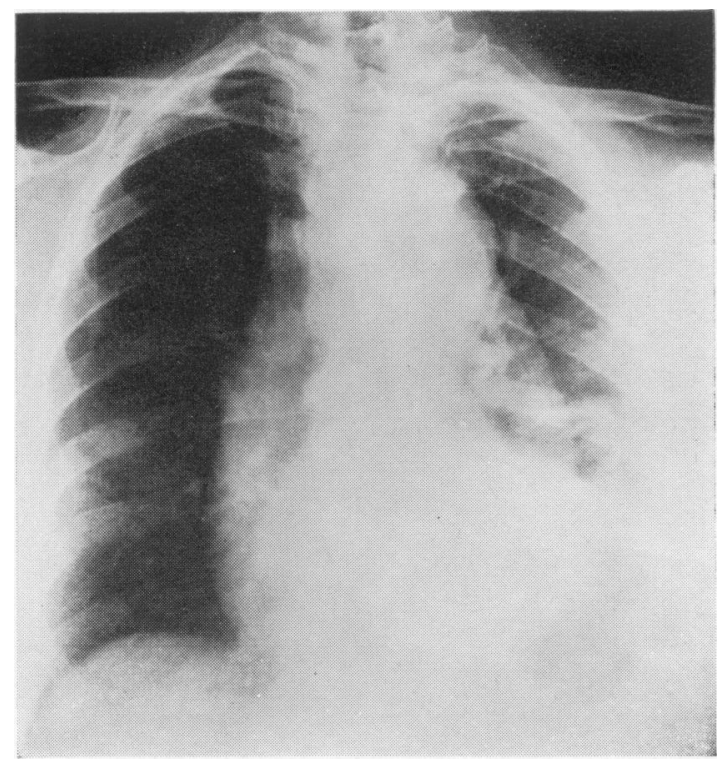

FiG. 1 


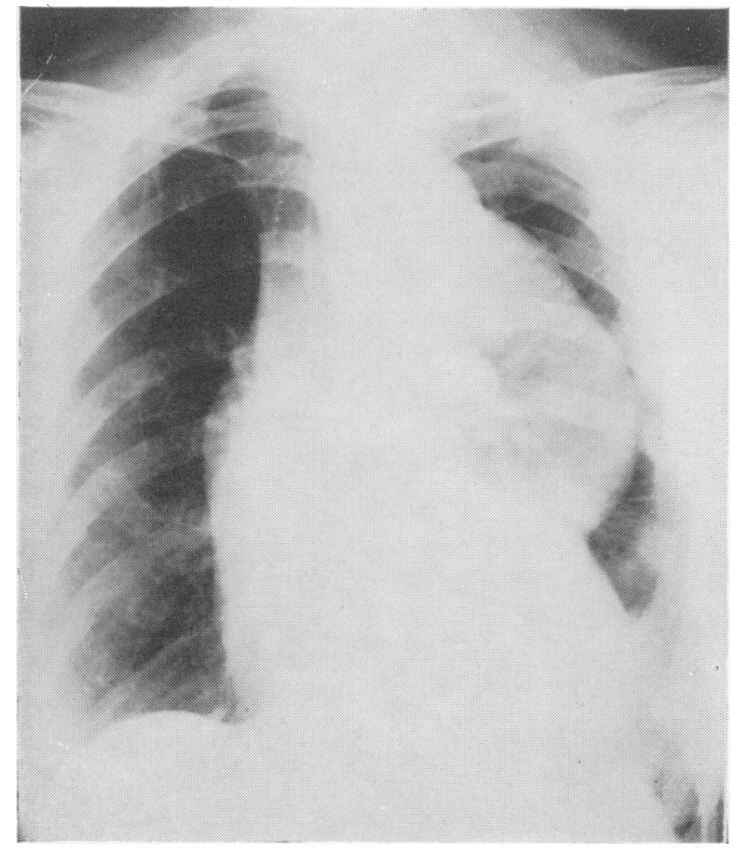

FiG. 2

after the initial pain, the other 10 weeks after. Both were quite severe and lasted for several hours.

Following this she was quite well and when seen in September 1966, 16 months after the initial rupture, she had no complaints and was able to get around the house by herself. On examination the blood pressure was 230/140 lying and standing and no other abnormal signs were found. There was no sign of extension of the aneurysm or obliteration of peripheral pulses, but her X-ray (Fig. 2) showed further dilatation of the aneurysm.

\section{Discussion}

There is no doubt that this patient ruptured a dissecting aneurysm into her left pleural cavity. It is likely that the initial rupture was a small one and sealed itself off as in Post's case. The recurrent attacks of chest pain may have been due to further dissection and re-entry of the aneurysm into the aorta and this would explain her survival for so long despite an enlarging aneurysm.

\section{Summary}

A case is described in which a 77-year-old woman remains alive and well 16 months after the intrapleural rupture of a dissecting aortic aneurysm.

\section{Acknowledgment}

I would like to thank Dr L. I. M. Castleden for his permission to publish this case.

\section{References}

Campbell, M. (1946) Dissecting aneurysm of aorta with survival for three months after rupture into pleura. Brit. Heart J. 8, 200.

Hirst, A.E., Johns, V.J. \& Kine, S.W. (1958) Dissecting aneurysm of the aorta. A review of 505 cases. Medicine (Baltimore), 37, 217.

Post, F. (1941) Haemothorax from leakage of a dissecting aortic aneurysm; survival. Lancet, ii, 558.

Sheenan, T. (1934) Spec. Rep. Ser. med. Res. Coun. Lond. 193. 